\title{
Shubnikov-de Haas oscillations in layered conductors with stacking faults
}

\author{
V. M. Gvozdikov \\ Department of Physics, Kharkov National University, 4 Svobody Sq., 61077 Kharkov, Ukraine; \\ Grenoble High Magnetic Field Laboratory MPI-FRF and CNRS, BP 166 F-38042, Grenoble Cedex 09, France \\ E-mail: Vladimir.M.Gvozdikov@univer.kharkov.ua
}

Received July 9, 2001

\begin{abstract}
The Shubnikov-de Haas ( $\mathrm{SdH}$ ) oscillations of the in-plane conductivity of layered 2D electron gas is calculated. It is shown that layer stacking faults, magnetoimpurity bound states, and electron scattering modulate the $\mathrm{SdH}$ oscillations via the specific factors which bear the structural information. At zero temperature the $2 \mathrm{D} \mathrm{SdH}$ oscillations are strongly nonsinusoidal in shape and related by simple equation to the derivative of the de Haas-van Alphen magnetization oscillations with respect to the magnetic field.
\end{abstract}

PACS: 71.10.Ca, 72.10.Fk, 73.61.-r

\section{Introduction}

The discovery of the high- $T_{c}$ superconductivity in layered cuprates as well as unconventional electronic properties of layered organic superconductors makes structural studies of these layered conductors of great interest. In particular, much of recent experimental effort has been devoted to the de Haasvan Alphen (dHvA) and the Shubnikov-de Haas ( $\mathrm{SdH})$ studies in organic superconductors [1-7]. Both the dHvA and $\mathrm{SdH}$ measurements in these quasi-two-dimensional (Q2D) materials displayed numerous deviations from the conventional threedimensional (3D) theory of Lifshits and Kosevich (LK) [8] many of which are remained yet not understood. For example, a sawtooth profile of the dHvA signal was predicted at zero temperature $T=0$ for purely $2 \mathrm{D}$ electron gas in the canonical ensemble (i.e., for the fixed number of electrons in the sample) [9] and an inverse sawtooth was found within the grand canonical ensemble (i.e., for the fixed chemical potential in the sample) [10]. In the first case the dHvA oscillations are due to the oscillations of the chemical potential $\mu$. The recent analysis of this matter given in the work [11] shows that only for low electron concentration when it is possible to realize experimentally a regime $\hbar \Omega \approx \mu$ ( $\Omega=e H / m c$ is the cyclotron frequency), oscillations of $\mu$ with inverse magnetic field $1 / H$ are important. In the case $\mu>>\hbar \Omega$ the oscillations of the chemical potential are small and one can use the grand canonical ensemble as it holds in the standard 3D LK theory.

Real layered conductors are far from being an ideal $2 \mathrm{D}$ electron gas. As a rule they consist of a stack of $2 \mathrm{D}$ conducting planes with anisotropic $2 \mathrm{D}$ Fermi surface within them and may contain impurities as well as some amount of stacking faults appearing as a result of the intercalation. The impact of the stacking faults on the dHvA oscillations in a layered conductors was studied in the work of the author [12]. It was shown that amplitudes of the dHvA harmonics are modulated by the layerfactor

$$
I_{p}=\int_{-\infty}^{\infty} N(\varepsilon) \exp \left(i \frac{2 \pi p \varepsilon}{\hbar \Omega}\right) d \varepsilon
$$

which is a Fourier-transform of the one-dimensional density of states (DOS) $N(\varepsilon)$ related to the electron hopping across the layers ( $p$ is the number of harmonic).

The delta peak in the $N(\varepsilon) \propto \delta\left(\varepsilon-\varepsilon_{0}\right)$ at some energy $\varepsilon_{0}$ makes $I_{p}$ to be an oscillatory function in the inverse field

$$
I_{p} \sim \cos \left(\frac{2 \pi p \varepsilon_{0}}{\hbar \Omega}\right)
$$


with the frequency determined by $\varepsilon_{0}$. Another modulation factors appear in the case of the magnetic field parallel to the layers [13] and under the conditions of the coherent magnetic breakdown [14].

Impurities play a significant role. In the theory of the dHvA oscillations they smear the Landau levels and thereby decrease the harmonic amplitudes via the Dingle factor [10], or they may create a bound electronic states which manifests themselves through beats of the dHvA oscillations [15] which are more prominent in the $2 \mathrm{D}$ case [16].

Contrary to the dHvA effect the $\mathrm{SdH}$ oscillations appear as a result of the electron life-time oscillations due to the scattering on impurities in external magnetic field [17]. Theoretically it is a much more difficult problem than the study of the thermodynamic oscillations and, consequently, the number of publications on the $\mathrm{SdH}$ effect in layered conductors is much less than on the dHvA effect in Q2D systems. The $\mathrm{SdH}$ effect in a $2 \mathrm{D}$ electron gas case was considered in a few works reviewed in the paper [18]. The anisotropy in a 2D model was considered numerically in the paper [19] in the context of the $\mathrm{SdH}$ studies of the organic superconductors. Longitudinal magnetoresistance quantum oscillations due to the coherent hopping across the layers has been numerically studied in Refs. 20 and 21 for the incoherent case, i.e., when $\tau>\hbar / t$ ( $\tau$ is the electron life-time, $t$ stands for the hopping integral between the neighboring layers).

In this paper we calculate $\tau$ in a layered $2 \mathrm{D}$ electron gas containing small amount of stacking faults in the perpendicular to layers external quantizing magnetic field and then apply the results of these calculations to the theoretical consideration of the $\mathrm{SdH}$ oscillations in this system.

\section{The life-time calculation}

A theory for the single-electron life-time $\tau(E)$ in a $2 \mathrm{D}$ electron gas due to the impurity scattering in an external quantizing magnetic field was developed by Ando and Uemura [22]. This theory provided a basis for further studies of different properties of a 2D electron gas in magnetic fields [18]. Maniv and Vagner [23] have generalize the approach by Ando and Uemura to the case of layered electron gas. These authors have shown that in layered conductors the interplane tunneling of electrons can significantly reduces the $2 \mathrm{D}$ scattering rate due to the possibility of escaping the impurity by hopping to the neighboring conducting planes. They calculated the magnetic field dependence of $\tau$ assuming the kinetic energy across the layers $\varepsilon\left(k_{z}\right)=k_{z}^{2} / 2 m$ to be limited within the band $0 \leq \varepsilon\left(k_{z}\right) \leq \Delta_{z}$. Here we generalize their approach to the case when the interlayer hopping energy is an arbitrary quantity distributed within the DOS $N(\varepsilon)$. Then the equation for the single electron self energy $\sigma(\omega)$ yields [23]:

$$
\sigma(\omega)=b_{0}+c_{0} \sum_{n=0}^{\infty} \int_{-\infty}^{\infty} d \varepsilon \frac{N(\varepsilon)}{\varepsilon-\gamma_{n}(\omega)+\sigma(\omega)}
$$

where

$$
\begin{gathered}
\gamma_{n}(\omega)=\omega-\hbar \Omega(n+1 / 2)+\mu, \\
b_{0}=U_{0} n_{i}, \quad c_{0}=\frac{U_{0}^{2} n_{i}}{4 \pi^{2} l_{H}^{2}},
\end{gathered}
$$

$U_{0}$ is the impurity potential amplitude $V(\mathbf{r})=$ $=U_{0} \delta\left(\mathbf{r}-\mathbf{r}_{j}\right), n_{i}$ is the impurity concentration, and $l_{H}=(\hbar c / e H)^{1 / 2}$ is the magnetic length.

Separating the real and imaginary parts in Eq. (2) for the self-energy $\sigma(\omega)=\operatorname{Re} \sigma(\omega)+i \operatorname{Im} \sigma(\omega)$, we have

$$
\begin{gathered}
\operatorname{Im} \sigma(\omega)=c_{0} \sum_{n=0}^{\infty} \int_{-\infty}^{\infty} d \varepsilon N(\varepsilon) \times \\
\times \frac{\operatorname{Im} \sigma(\omega)}{\left[\gamma_{n}(\omega)-\operatorname{Re} \sigma(\omega)-\varepsilon\right]^{2}+\operatorname{Im}^{2} \sigma(\omega)} .
\end{gathered}
$$

Since we are interested in quantum oscillations which holds in the long life-time regime, $\hbar / \tau(\omega)=\operatorname{Im} \sigma(\omega)<<\hbar \Omega$, one can neg lect the quantity $\operatorname{Re} \sigma(\omega) \simeq U_{0} n_{i}<<\mu$ as a small correction to the chemical potential $\mu$.

Applying then a standard summation procedure $[10,17]$ to Eq. (5) we can recast it into a form

$$
\begin{aligned}
\operatorname{Im} \sigma(\omega)=a & \left\{1+2 \operatorname{Re} \sum_{p=1}^{\infty}(-1)^{p} \exp \left[-\frac{2 \pi \operatorname{Im} \sigma(\omega)}{\hbar \Omega}\right] \times\right. \\
& \left.\times I_{p} \cos \left[2 \pi\left(\frac{\omega}{\Omega}+\delta\right) p\right]\right\}
\end{aligned}
$$

where $a=n_{i} E_{0} / 2 \pi$ with $E_{0}=m U_{0}^{2} / 2 \hbar^{2} \quad$ being the resonance energy for the $\delta$-potential scattering and $\delta=\Delta[1 / H] / \hbar \Omega$ is a small phase shift $0<$ $<\Delta[1 / H]<1 / 2$ arising due to that the ratio 
$\mu / \hbar \Omega$ is not exactly the integer: $\mu / \hbar \Omega=N+$ $+\Delta[1 / H]$ ( $N$ stands for integer $)$. The factor $I_{p}$ here is given by Eq. (1). This factor was studied in detail in the paper [12] devoted to the dHvA effect in layered conductors. For periodic layered system with the nearest layer hopping $N(\varepsilon)=1 / \pi\left(4 t^{2}-\varepsilon^{2}\right)^{1 / 2}$ and

$$
I_{p}=J_{0}\left(\frac{4 \pi t p}{\hbar \Omega}\right),
$$

where $J_{0}(x)$ is the Bessel function, $t$ is the interlayer hopping integral.

In case when there is a small concentration $c \ll 1$ of the layer stacking faults the factor $I_{p}$ was calculated in [12] to yield

$$
I_{p}=(1-c) J_{0}\left(\frac{4 \pi t p}{\hbar \Omega}\right)+c \delta I_{p}
$$

where

$$
\delta I_{p}=\exp \left(-\frac{2 \pi p}{\hbar \Omega} \sqrt{t^{2}-t_{0}^{2}}\right) \cos \left(\frac{4 \pi t_{0}}{\hbar \Omega} p\right) .
$$

The stacking fault means that the local hopping integral $t_{0}$ relevant to the electron transition from the «defect layer» to the nearest neighbor layers is less than $t$. Physically this kind of stacking faults may appear as a result of the intercalation of the layer conductor.

\section{Shubnikov-de Haas oscillations}

Consider now the SdH oscillations in a stack of $2 \mathrm{D}$ isotropic conductors with impurities in the perpendicular magnetic field. We do not assume periodicity in the layer stacking. The in-plane conductivity tensor diagonal elements, therefore, are given by [18]

$$
\sigma_{\alpha \alpha}=-\frac{N_{e} e^{2}}{m} \int_{0}^{\infty} d E\left(\frac{\partial f}{\partial E}\right) \tau(E),
$$

where $N_{e}$ is the 2D density of conducting electrons within the layer and $f(E)$ is the Fermi function.

The energy-dependent life-time $\tau(E)=\hbar / \operatorname{Im} \sigma(E)$ can be found from Eq. (6) in the first approximation on the parameter $a / \hbar \Omega<<1$ which yields

$$
\tau(E) \approx \frac{\hbar}{a}\left\{1-2 \sum_{p=1}^{\infty}(-1)^{p} \exp \left(-\frac{2 \pi p a}{\hbar \Omega}\right) \times\right.
$$

$$
\left.\times I_{p} \cos \left[2 \pi\left(\frac{E}{\hbar \Omega}+\delta\right) p\right]\right\} .
$$

Substituting Eq. (11) into Eq. (10) and completing the standard integration, we have

$$
\sigma_{\alpha \alpha}=\sigma_{0}+\tilde{\sigma}
$$

where $\sigma_{0}=N_{e} e^{2} \tau_{0} / m$ is the smooth part of the conductivity with $\tau_{0}=\hbar / a=4 \pi^{2} \hbar^{3} / m U_{0}^{2} n_{i}$.

The oscillating part of the conductivity $\tilde{\sigma}$ is given by

$$
\tilde{\sigma}=-2 \sigma_{0} \sum_{p=1}^{\infty}(-1)^{p} \exp \left(-\frac{2 \pi p}{\tau_{0} \Omega}\right) R_{p}^{T} I_{p} \cos \left(\frac{2 \pi \mu}{\hbar \Omega} p\right)
$$

where

$$
R_{p}^{T}=\frac{Z_{p}}{\sinh Z_{p}}
$$

is the standard temperature factor with $Z_{p}=$ $=2 \pi^{2} T p / \hbar \Omega$. We also have neglected the small phase shift $\delta$ since energies of the order of $E \simeq \mu$ are essential in the integral of Eq. (10).

Considering at first the case of a solitary layer $\left(I_{p}=1\right)$ and zero temperature $\left(R_{p}^{T}=1\right)$ one can perform an elementary summation in Eq. (14) to obtain

$$
\tilde{\sigma}=\sigma_{0}\left[1-\frac{\sinh v}{\cosh v+\cos \left(\frac{2 \pi \mu}{\hbar \Omega}\right)}\right] \text {, }
$$

where $v=2 \pi / \Omega \tau_{0}$

On the other hand, the oscillating part of the magnetization of a $2 \mathrm{D}$ electron gas at zero temperature can be written as a sum [10]

$$
\tilde{M}=M_{0} \sum_{p=1}^{\infty} \frac{(-1)^{p}}{p} \exp \left(-\frac{2 \pi p}{\tau_{0} \Omega}\right) \sin \left(\frac{2 \pi \mu}{\hbar \Omega} p\right)
$$

which can be completed to yield

$$
\tilde{M}=M_{0} \arctan \left[\frac{\sin \left(\frac{2 \pi \mu}{\hbar \Omega}\right)}{\cos \left(\frac{2 \pi \mu}{\hbar \Omega}\right)+e^{\nu}}\right] .
$$

Here $M_{0}$ is independent of the magnetic field $H$. 
Comparing Eqs. (15) and (17) we arrive at the relation between $\tilde{\sigma}$ and $\tilde{M}$ for $2 \mathrm{D}$ electron gas with the $\delta$-potential impurities at zero temperature:

$$
\frac{\tilde{\sigma}}{\sigma_{0}} \approx A H^{2} \frac{\partial}{\partial H}\left(\frac{\tilde{M}}{M_{0}}\right),
$$

where $A=e \hbar / \pi m c \mu$. Since $A H \simeq \hbar \Omega / \pi \mu \ll 1$, we see that relative amplitude of the $\mathrm{SdH}$ oscillation $\tilde{\sigma} / \sigma_{0}$ is much less than the corresponding relative oscillations of the magnetization $\tilde{M} / M_{0}$ in the dHvA effect. Nonetheless, the first observation in 1930 of the $\mathrm{SdH}$ oscillations by Shubnikov and de Haas preceded the discovery of the dHvA effect [24].

Recently, Eq. (18) was proved experimentally in a 2D organic superconductor of ET-family [7]. The shape of the $\mathrm{SdH}$ oscillations observed was nonsinusoidal and very similar to that given by Eq. (15) and shown in Fig. 1. What is strange in this connection that the SdH signal was measured in the geometry when both the magnetic field and current
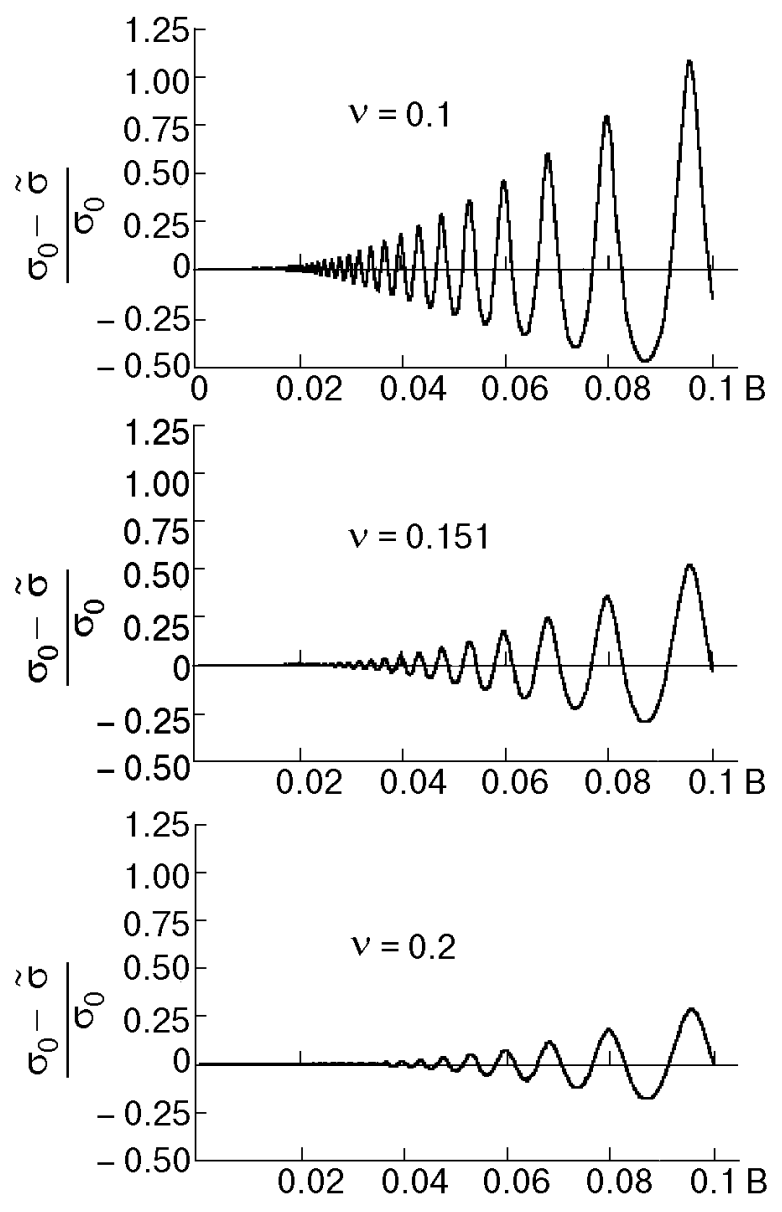

Fig. 1. Field dependence of the conductivity given by Eq. (15) (in conventional units $B=\hbar \Omega$ ) for $\mu=1000, v=0.1,0.151,0.2$ $\left(v=2 \pi \hbar / \tau_{0}\right)$. have been directed perpendicular to layers. The temperature was nonzero and varied within the range $0.4-1.27 \mathrm{~K}$. On the other hand, it is easy to see that, strictly speaking, Eq. (18) does not hold under this conditions. First of all this is because of the anisotropy of the conductivity in the direction perpendicular to layers. Secondly, the layer-factor $I_{p}$, as well as the factor $R_{p}^{T}$ (as well as some other factors which we discuss below) are functions of the inverse magnetic field $1 / H$ and this must be taken into account in the derivative $\partial \tilde{M} / \partial H$.

An additional degrees of freedom related to the spin of electron and bound states due to impurities (the so-called magnetoimpurity levels [15]) can be easily taken into consideration. In these cases the energy of an electron gains a shift $\varepsilon \rightarrow \varepsilon+\zeta$, where $\zeta$ is an energy variable related to the additional degree of freedom. If the DOS for $\zeta$ is given by $G(\zeta)$, then

$$
I_{p} \rightarrow I_{p} R_{p}
$$

where

$$
R_{p}=\int_{-\infty}^{\infty} d \zeta G(\zeta) \exp \left(i \frac{2 \pi \zeta}{\hbar \Omega} p\right)
$$

In the case of a spin

$$
G(\zeta)=\frac{1}{2}\left[\delta\left(\zeta-\mu_{e} H\right)+\delta\left(\zeta+\mu_{e} H\right)\right]
$$

where $\mu_{e}=\hbar e / 2 m_{e} c$ is the Bohr magneton. The corresponding spin-factor $R_{p}^{s}$ reads

$$
R_{p}^{s}=\cos \left(\pi \frac{m}{m_{e}} p\right) .
$$

This is a standard spin-factor, known in magnetooscillations [10]. Another factor appears if impurities split off bound states from the Landau levels [15]. This magnetoimpurity effect is more strong in 2D systems than in a $3 \mathrm{D}$ case. If we denote this spliting by $\Delta_{i}(H)$, then the appropriate DOS is again given by the $\delta$-function

$$
G^{i}(\zeta)=\delta\left(\zeta \pm \Delta_{i}\right)
$$

The corresponding damping factor due to the magnetoimpurity bound states is

$$
R_{p}^{i}=\cos \left(\frac{2 \pi \Delta_{i}}{\hbar \Omega} p\right) .
$$


Signs \pm in Eq. (23) correspond to attracting and repulsing impurities. In the case $\Delta_{i}<<\hbar \Omega$ the splitting value does not depend on $p$ [16]. Putting together all the above discussed damping factors and taking $I_{p}$ factor in the form given by Eqs. (7)-(9), we can write the oscillating part of the in-plane conductivity in the form

$$
\tilde{\sigma}=\tilde{\sigma}_{1}+\tilde{\sigma}_{2}
$$

with

$$
\begin{gathered}
\tilde{\sigma}_{1}=-2 \sigma_{0}(1-c) \sum_{p=1} \exp \left(-\frac{2 \pi p}{\tau_{0} \Omega}\right) \times \\
\times R_{p}^{T} R_{p}^{i} R_{p}^{s} J_{0}\left(\frac{4 \pi t}{\hbar \Omega} p\right) \cos \left(\frac{2 \pi \mu}{\hbar \Omega} p\right) \\
\tilde{\sigma}_{2}=-2 \sigma_{0} c \sum_{p=1} \exp \left(-\frac{2 \pi p}{\tau_{1} \Omega}\right) \times \\
\times R_{p}^{T} R_{p}^{i} R_{p}^{s} \cos \left(\frac{4 \pi t}{\hbar \Omega} p\right) \cos \left(\frac{2 \pi \mu}{\hbar \Omega} p\right) .
\end{gathered}
$$

Here $v_{1}=2 \pi / \tau_{1} \Omega$, where

$$
\tau_{1}=\frac{\hbar}{a+\sqrt{t^{2}-t_{0}^{2}}}
$$

is the renormalized due to the stacking faults effective electron life-time. At zero temperature we have

$$
\begin{gathered}
\tilde{\sigma}_{2}=-\frac{\sigma_{0} c}{8} \times \\
\times \sum_{\alpha, \beta, \gamma= \pm}\left\{\frac{\sinh v_{1}}{\cosh v_{1}-\cos \left[\left(\mu+\alpha \Delta_{i}+\beta t\right)+\gamma \frac{\pi m}{m_{e}}\right]}-1\right\} .
\end{gathered}
$$

Despite that $\tilde{\sigma}_{2}$ is proportional to the stacking fault concentration $c$ it may be essential for small $v_{1} \ll 1$ since in this case $\tilde{\sigma}_{2}$ has a sharp $\delta$-like peaks, as it is seen in Fig. 2.

\section{Conclusions and discussion}

The two major experimental tools of the fermiology, the SdH and the dHvA effects, proved to be very useful not only for studies of conventional 3D metals but as well for recent analysis of the new layered organic conductors and superconductors in-
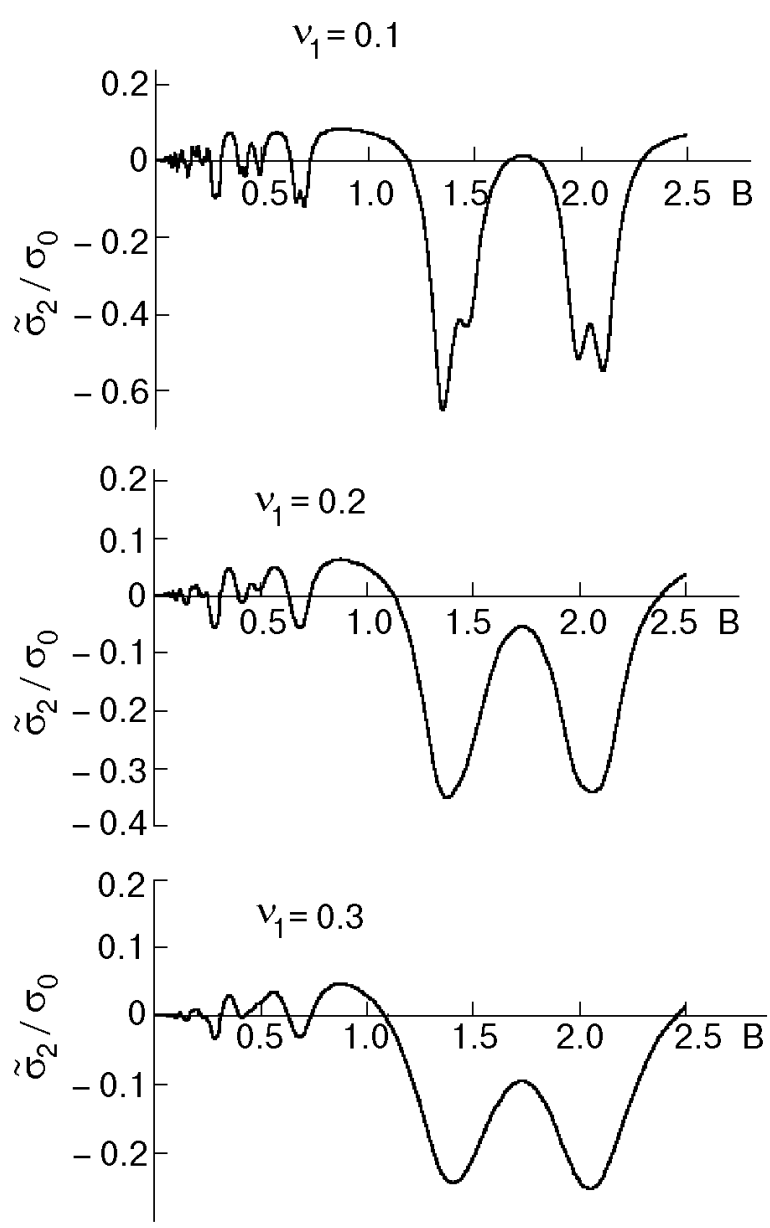

Fig. 2. Field dependence of the oscillating part of the conductivity caused by the stacking faults and given by Eq. (29) (in conventional units $B=\hbar \Omega$ ) for $\mu=1000, c=0.1, \quad t=0.1$, $\Delta_{i}=0.5, m / m_{e}=1.5, v_{1}=0.1,0.2,0.3\left(v_{1}=2 \pi \hbar / \tau_{1}\right)$.

cluding the high- $T_{c}$ cuprates. Though both effects are based on the Landau quantization, the $\mathrm{SdH}$ effect, as it is well known from the textbooks on the fermiology $[10,17,25]$, is much more difficult to study theoretically since it requires calculations for the transition probabilities caused by the electron scattering on impurities in external magnetic field. This scattering in its turn strongly depends on the dimensionality of the system in question and manifests itself in different kinetic characteristics of organic conductors, such as the high frequency impedance [26], and the SdH in quasi-2D conductors which is the subject of the present publication. Contrary to the common-spread opinion, even among the experts, it is the $\mathrm{SdH}$ oscillations, rather than the dHvA effect, have been observed first in 1930 in Leiden by Shubnikov and de Haas and thereby laid the foundation to the modern fermiology. This point as well as the dramatic history of 
the discovery of the $\mathrm{SdH}$ effect one can find in the interesting historical essay [24].

In this paper we have calculated the $\mathrm{SdH}$ oscillations within the approach which generalizes the Green function method developed by Maniv and Vagner [23] for the electron scattering problem in a quasi-2D electron gas. The calculated oscillating part of the in-plane conductivity tensor diagonal component $\tilde{\sigma}$, Eq. (13), as well as the electron life-time $\tau$, Eq. (11), turned out to be dependent on the very same layer factor $I_{p}$, Eq. (1), which modulates the dHvA oscillations of the layered conductors [12] and depends on the layer stacking. At zero temperature and in the $2 \mathrm{D}$ limit a simple relation was established between the relative inplane $\mathrm{SdH}$ oscillations, $\tilde{\sigma} / \sigma_{0}$, and the corresponding relative $\mathrm{dHvA}$ oscillations of the magnetization $\tilde{M} / M_{0}$. This relation is given by Eq. (18) which holds only for the $2 \mathrm{D}$ electron gas. The anisotropy of the 2D Fermi surface, as well as nonzero temperature and electron hopping across the layers bring additional terms to the Eq. (18). Nonetheless, this equation was experimentally verified by the simultaneous dHvA and $\mathrm{SdH}$ measurements on the $\beta^{\prime \prime}-(\mathrm{BEDT}-\mathrm{TTF})_{2} \mathrm{SF}_{5} \mathrm{CH}_{2} \mathrm{SO}_{3}$ organic conductor within the temperature range $0.4-1.27 \mathrm{~K}$ for the interlayer component of the conductivity tensor [7]. The shape of these strongly nonsinusoidal oscillations is the same as given by Eq. (15) and shown in Fig. 1. The enhancement of the impurity scattering potential makes these oscillations less in the amplitude but more sine-like in the form. The spin-splitting and magnetoimpurity bound states yield an additional factors, $R_{p}^{S}$ and $R_{p}^{i}$ correspondingly, which modulates the $\mathrm{SdH}$ oscillations (see Eqs. (25) $-(27)$ ) and result in a big variety of the oscillation patterns as it is displayed in Figs. 2 and 3.

The stacking faults modify the Dingle-like exponent in Eq. (27) through the effective life-time $\tau_{1}$ (Eq. (28)). The appropriate correction to the oscillation $\tilde{\sigma}_{2}$ arises due to the stacking faults and has at zero temperature a periodic $\delta$-like peaks when $v_{1} \ll 1$. It is essential even for low concentration of the stacking faults, as one can see in Fig. 2.

The oscillation patterns displayed in Figs. 1-3 are very diverse. The peaks in Figs. 2, 3 are splitted due to the spin and magnetoimpurity factors and sensitive to variations of the other parameters of the model. Some additional analysis of the SdH oscillations within this model will be published elsewhere. The results and methods of this paper will be also applied to an anisotropic 2D Fermi surfaces structures typical for real organic layered (super)conductors.
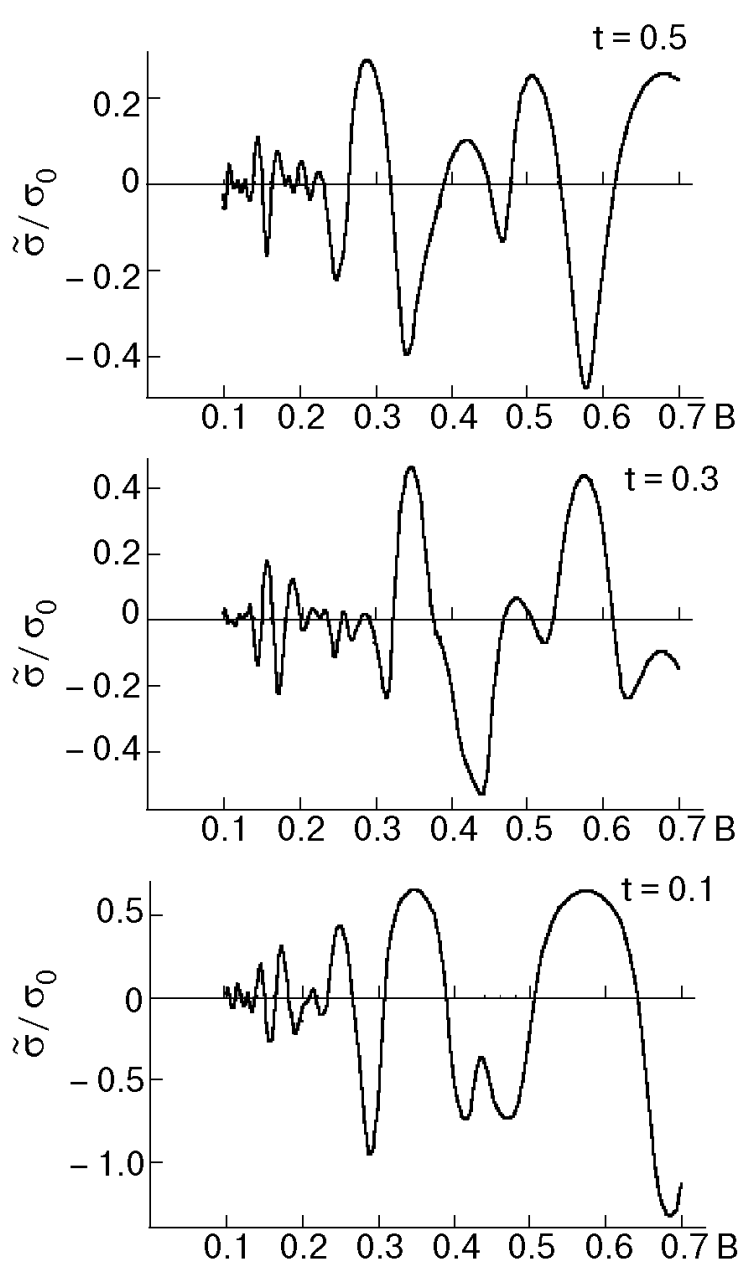

Fig. 3 Field dependence of the oscillating part of the conductivity given by Eq. (25)-(28) (in conventional units $B=\hbar \Omega$ ) for $\mu=1000, c=0.2, \Delta_{i}=0.5, m / m_{e}=1.5, T=0.001, v=0.1$, $v_{1}=0.2, t=0.1,0.3,0.5\left(v=2 \pi \hbar / \tau_{0}, v_{1}=2 \pi \hbar / \tau_{1}\right)$.

\section{Acknowledgments}

The author is grateful to Tsofar Maniv and Israel Vagner for numerous stimulating discussions on the problem of electron scattering in a quasi-2D gas in magnetic field and to Peter Wyder for the hospitality during his stay in the Grenoble High Magnetic Field Lab.

1. F. A. Meyer, E. Steep, W. Biberacher, P. Crist, A. Lerf, A. G. M. Jansen, W. Joss, and P. Wyder, Europhys. Lett. 32, 681 (1995).

2. N. Harrison, J. Caulfeld, J. Singleton, P. H. P. Reinders, F. Herlach, W. Hayes, M. Kurmoo, and P. Day, J. Phys. Cond. Matter 8, 5415 (1996).

3. E. Steep,L. H. Nguen, W. Biberacher, H. Muller, A. G. M. Jansen, and P. Wyder, Physica B259-261,1079 (1999).

4. M. M. Honold, N. Harrison, M. S. Nam, J. Singleton, C. H. Mielke, M. Kurmoo, and P. Day, Phys. Rev. B58, 7560 (1998). 
5. M. Shiller, W. Schmidt, E. Balthes, D. Schwitzer, H. J. Koo, M. H. Wanhbo, I. Heinen, T. Kalusa, P. Kircher, and W. Strunz, Europhys. Lett. 51, 82 (2000).

6. M. V. Kartsovnik, G. Yu. Logvenov, T. Ishiguro, W. Biberacher, H. Anzai, and N. D. Kushch, Phys. Rev. Lett. 77, 2530 (1996).

7. J. Wosnitza, S. Wanka, J. Hagel, H. v. Lohneysen, J. S. Qualls, J. S. Brooks, E. Balthes, J. A. Schlueter, U. Geiser, J. Mohtasham, R. W. Winter, and G. R. Gard, Phys. Rev. Lett. 86, 508 (2001).

8. I. M. Lifshitz and A. M. Kosevich, Sov. Phys. JETP 2, 639 (1953).

9. I. D. Vagner, T. Maniv, and E. Ehrenfreund, Phys. Rev. Lett. 51, 1700 (1983); K. Jauregui, V. I. Marchenko, and I. D. Vagner, Phys. Rev. B41, 12922 (1990); P. D. Grigor'ev and I. D. Vagner, Pis'ma Zh. Exp. Teor. Fiz. 69, 139 (1999).

10. D. Shoenberg, Magnetic Oscillations in Metals, Cambridge University Press, Cambridge (1984).

11. T. Champel and V. P. Mineev, Philos. Mag. B81, 55 (2001).

12. V. M. Gvozdikov, Sov. Phys. Solid State 26, 1560 (1984).

13. V. M. Gvozdikov, Sov. Phys. Solid State 28, 179 (1986).

14. V. M. Gvozdikov, Fiz. Nizk. Temp. 12, 705 (1986) [Sov. J. Low Temp. Phys. 12, 399 (1986)].
15. A. M. Ermolaev and M. I. Kaganov, Pis'ma Zh. Exp. Teor. Fiz. 6, 984 (1967).

16. V. M. Gvozdikov, A. M. Ermolaev, and Yu. A. Sharapov, Vestnik Kharkovskogo Natzional'nogo Universiteta, № 476, Ser. «Fizika», N 4, 18 (2000).

17. A. A. Abrikosov, Fundamentals of Theory of Metals, Nauka, Moskow (1987) (in Russian).

18. T. Ando, A. B. Fowler, and F. Stern, Rev. Mod. Phys. 54, 437 (1982).

19. N. Harrison, R. Bogaerts, P. H. P. Reinders, J. Singleton, S. J. Blundell, and F. Herlach, Phys. Rev. B54, 9977 (1996).

20. A. E. Datars and J. E. Sipe, Phys. Rev. B51, 4312 (1995).

21. R. H. Mc Kenzie and P. Moses, Phys. Rev. Lett. 81, 44922 (1998).

22. T. Ando and Y. Uemura, J. Phys. Soc. Jpn. 36, 959 (1974).

23. T. Maniv and I. D. Vagner, Phys. Rev. B38, 6301 (1988).

24. B. I. Verkin, S. A. Gredeskul, L. I. Pastur, and Yu. A. Freyman, Fiz. Nizk. Temp. 16, 1203 (1990) [Sov. J. Low. Temp. Phys. 16, 696 (1990)].

25. I. M. Lifshits, M. Ya. Azbel', and M. I. Kaganov, The Electron Theory of Metals, Nauka, Moskow (1971).

26. V. G. Peschansky, I. V. Kozlov, and K. Jiasemides, Fiz. Nizk. Temp. 26, 225 (2000) [Low Temp. Phys. 26, 169 (2000)]. 